EPJ Web of Conferences 70, 00055 (2014)

DOI: 10.1051/epjconf/20147000055

(C) Owned by the authors, published by EDP Sciences, 2014

\title{
The ICARUS Expriment at LNGS Underground Laboratory
}

\author{
Nicola Canci $^{\mathrm{a}}$ on behalf of the ICARUS Collaboration \\ ${ }^{1}$ INFN - Laboratori Nazionali del Garn Sasso \\ S.S 17 bis, $\mathrm{km} \mathrm{18+910}$ \\ Assergi (AQ), Italy
}

\begin{abstract}
ICARUS (Imaging Cosmic And Rare Underground Signals) is, so far, the largest Liquid Argon Time Projection Chamber (LAr-TPC containing 600 tons of LAr) addressed to the study of "rare events" and, among them, neutrino interactions.

Installed and operating underground, at the Gran Sasso National Laboratory (INFNLNGS, Italy), ICARUS started working gradually since summer 2010, collecting events from both, the cosmic rays able to reach the depth of the laboratory, and from CNGS neutrino beam.

The detector, providing a completely uniform imaging and calorimetry with a high accuracy on massive volumes, allows for complete event reconstruction.

The detection technique, as well as detector main features and performances are here described.

The recent measurement of the velocity of neutrinos with short bunched CNGS beam, consistent with the speed of light, and the search for the analogue to Cherenkov radiation effect for superluminal neutrinos are presented.
\end{abstract}

\section{Introduction}

The Liquid Argon Time Projection Chamber (LAr-TPC), first proposed by C. Rubbia in 1977, is a powerful detection technique providing a 3D imaging of any ionizing event [1].

The operational principle of the LAr TPC is based on the fact that a charged particle interaction in LAr induces atomic ionization accompanied by light emission in the VUV (128 nm) range.

In highly purified LAr ionization tracks can be transported practically undistorted by a uniform electric field (typically $500 \mathrm{~V} / \mathrm{cm}$ ) over macroscopic distances: in fact, the low diffusion coefficient allows to have undistorted tracks for $1.5 \mathrm{~m}$ at least [2], [3], [4]. Imaging is provided by a suitable set of electrodes (wires) placed at the end of the drift path continuously sensing and recording the signals induced by the drifting electrons [5] (see Fig. 1).

Non-destructive read-out of ionization electrons by charge induction allows to detect the signal of electrons crossing subsequent wire planes with different orientation. This provides several projective views of the same event, hence allowing space point reconstruction and precise calorimetric measurement.

In particular, the measurement of the absolute time of an ionizing event, combined with the electron drift velocity information $\left(\mathrm{v}_{D} \sim 1.6 \mathrm{~mm} / \mu \mathrm{s}\right.$ at $\left.\mathrm{E}_{D}=500 \mathrm{~V} / \mathrm{cm}\right)$, provides the absolute position of

\footnotetext{
a e-mail: nicola.canci@lngs.infn.it
} 
the track along the drift coordinate. The determination of the absolute time of the ionizing event is accomplished by the detection of the prompt scintillation light also exploited for trigger purposes [4].

This continuously sensitive and self triggering detector is characterized by high spacial resolution and granularity allowing for a precise reconstruction of event's topology. The event reconstruction is completed by calorimetric measurement via $d E / d x$ ionization signal over a very wide energy range, from $\mathrm{MeV}$ to several tens of $\mathrm{GeV}$.

Electrons are identified by the characteristic electromagnetic showering; they can be well separated from $\pi^{0}$ via $\gamma$ reconstruction, $d E / d x$ signal comparison in the first centimeters of shower and $\pi^{0}$ invariant mass measurement at the level of permil: this feature guarantees a $90 \%$ efficiency identification of the leading electron in $v_{e}$ charged-current $(\mathrm{CC})$ interactions, while rejecting neutral-current (NC) background to a negligible level [6]. Estimated energy resolutions for electromagnetic showers, hadronic showers and low energy electrons are reported in Tab. 1.

For long muon tracks escaping the detector, momentum is determined exploiting their multiple scattering by a Kalman filter algorithm with an average resolution $\Delta p / p \sim 15 \div 20 \%$, mainly depending on the track length [7].

Table 1. Estimated energy resolutions for electromagnetic showers, hadronic showers and low energy electrons.

\begin{tabular}{lr}
\hline Events & $\sigma(E) / E$ \\
\hline Electromagnetic Showers & $3 \% / \sqrt{E(G e V)}$ \\
Hadronic Showers & $30 \% / \sqrt{E(G e V)}$ \\
Low Energy Electrons & $(11 \% / \sqrt{E(M e V)})+2 \%$ \\
\hline
\end{tabular}

Figure 1. Illustration of the LAr-TPC working principle: from a charged particle ionization path in LAr to its geometrical reconstruction.

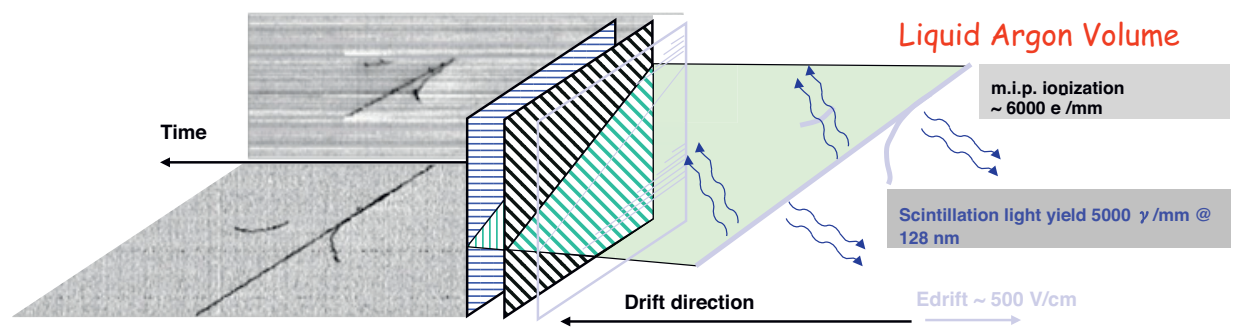

\section{The ICARUS T600 experiment}

\subsection{Detector overivew}

The ICARUS T600 detector consists of a large cryostat split into two identical, adjacent half-modules, with an overall volume of about 760 tons of ultra-pure liquid Argon at $89 \mathrm{~K}$ temperature (Fig. 2) [8]. Each half-module, with internal dimensions $3.6 \times 3.9 \times 19.6 \mathrm{~m}^{3}$, houses two Time Projection Chambers (TPC) sharing a common cathode. Each TPC is made of three parallel wire planes, $3 \mathrm{~mm}$ apart, oriented at $0^{\circ}$ and $\pm 60^{\circ}$ w.r.t. the horizontal direction: in total 53248 wires, with length up to $9 \mathrm{~m}$, are 
installed. By appropriate voltage biasing, the first two planes (Induction-1 and Induction-2 planes) are transparent to drift electrons and measure them in a non-destructive way, whereas the ionization charge is finally collected by the last one (Collection plane).

The application of an electric field $\mathrm{E}_{D}=500 \mathrm{~V} / \mathrm{cm}$, kept uniform by appropriate field shaping electrodes, ensures that the $1.5 \mathrm{~m}$ maximum drift distance is covered in $1 \mathrm{~ms}$. The signals coming from each wire are continuously read and digitized at $25 \mathrm{MHz}$ ( $\sim 400 \mathrm{~ns}$ t-sample) and recorded in multievent circular buffers. Photomultiplier Tubes (PMTs), operating at LAr temperature ${ }^{1}$, installed behind the wire planes are used to detect the prompt scintillation light and to determine the absolute time of the ionizing events and obtain the trigger signal.

Figure 2. Scheme of the detector [Left] and a picture of the LAr TPC with an artistic view of the working principle of one of the modules [Right].
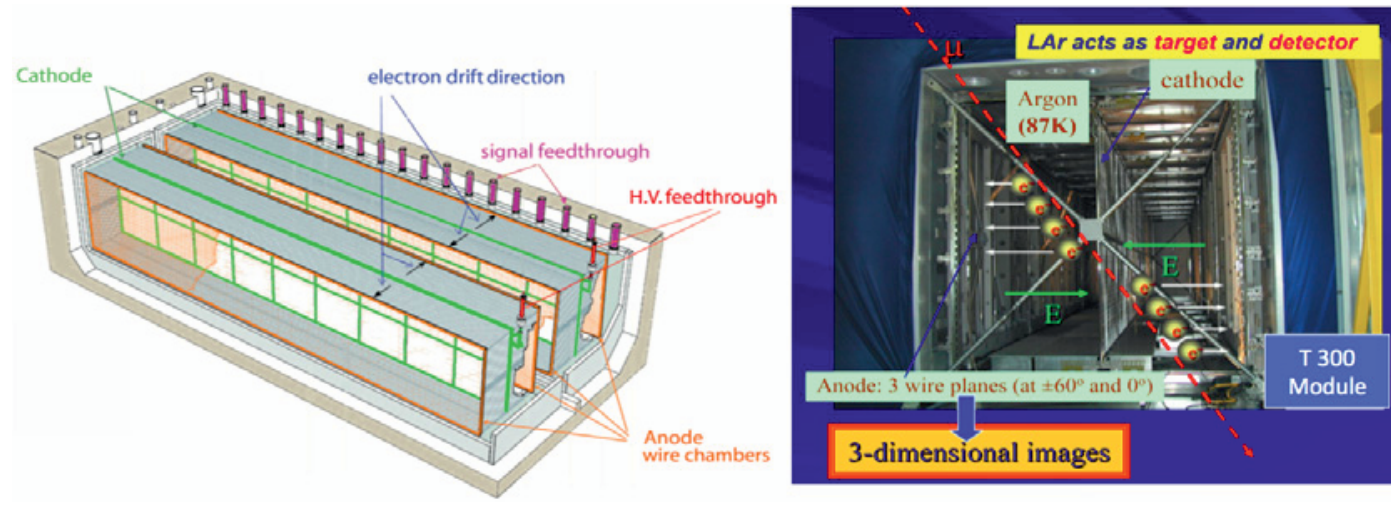

\subsection{Cryogenic plant}

The two half modules containing the LAr-TPC chambers are surrounded by a unique thermal insulation: it is realized by evacuated honeycomb panels assembled to realize a tight containment vessel [8]. Between the insulation and the aluminum containers a thermal shield is placed, with boiling Nitrogen circulating inside, to intercept the heat load and maintain the cryostat bulk temperature uniform (within $1 \mathrm{~K}$ ) and stable at $89 \mathrm{~K}$. Nitrogen used to cool the T600 half-modules is stored in two $30 \mathrm{~m}^{3}$ $\mathrm{LN}_{2}$ tanks positioned at the top of the plant. Its temperature is fixed by the equilibrium pressure in the tanks ( 2.1 bar, corresponding to about $84 \mathrm{~K})$, which is kept stable in steady state by a dedicated re-liquefaction system of 12 cryocoolers ( $48 \mathrm{~kW}$ global cold power), thus guaranteeing safe operation in closed-loop.

\subsection{Liquid Argon purity and purification}

A fundamental requirement for the performance of the liquid Argon TPC is that electrons produced by ionizing particles can travel unperturbed from the point of production to the collecting planes.

Tiny fractions of impurities $\left(\mathrm{O}_{2}, \mathrm{H}_{2} \mathrm{O}, \mathrm{CO}_{2}\right.$ and/or fluorinated-chlorinated compounds) diluted at $\sim 1$ parts per million (ppm) level in LAr are usually reported in commercially available Argon (best grade), due to the industrial process of air separation. These electronegative impurities may impair

\footnotetext{
${ }^{1}$ PMTs are made sensible to VUV scintillation light $(128 \mathrm{~nm})$ by applying a wavelength shifter layer (TPB).
} 
the detector performance by significantly reducing the amount of the charge available from ionization events in $\mathrm{LAr}^{2}$. To achieve long electron drift paths and to avoid the decrease the electron collection efficiency impurities in the liquid must be reduced to a very low level: in fact, to reach a negligible attenuation over the full drift distance, the concentration of impurities must be kept at the level of less than 0.1 part per billion ( $\mathrm{ppb}$ ) Oxygen equivalent. In addition, to ensure stable performance of the device, the purity must be preserved in the dewar hosting the inner detector for the longest possible period of time.

To this purpose, each half-module is equipped with two gas Argon and one liquid Argon recirculation/purification systems. Argon gas is continuously drawn from the cryostat ceiling and, recondensed, drops into Oxisorb ${ }^{T M}$ filters to finally return to the LAr containers [1].

The electron lifetime is directly related to the impurity concentration by an inverse linear relationship [9], [1] and depends on the drift field applied to the LAr active volume. The lifetime estimation thus provides a direct measurement of the LAr impurity content and on other hands, its value is used to correct the electron charge lost during the drift path in the tracks reconstruction.

To this aim, the electron lifetime is continuously monitored studying the attenuation of the charge signal as a function of the drift time along "clean" through-going muon tracks in Collection view, i.e. straight tracks without clear $\delta$-rays and associated $\gamma$ 's; the negative signal induced by the PMTs on the wires marks the time at which the track entered the detector. About 50 muon tracks are sufficient to daily measure the electron charge attenuation within a $3 \%$ precision, dominated by residual Landau charge fluctuations.

With the liquid recirculation turned on, the LAr purity steadily increased, reaching values of free electron lifetime $\left(\tau_{e}\right)$ exceeding $6 \mathrm{~ms}$ in both half-modules after few months of operation (Fig. 3). This corresponds to $0.05 \mathrm{ppb}_{2}$ equivalent impurity concentration, producing a maximum $16 \%$ charge attenuation, at the maximum $1.5 \mathrm{~m}$ drift distance.

Figure 3. Liquid Argon purity measurements for both the East [Left] and West [Right] cryostats.
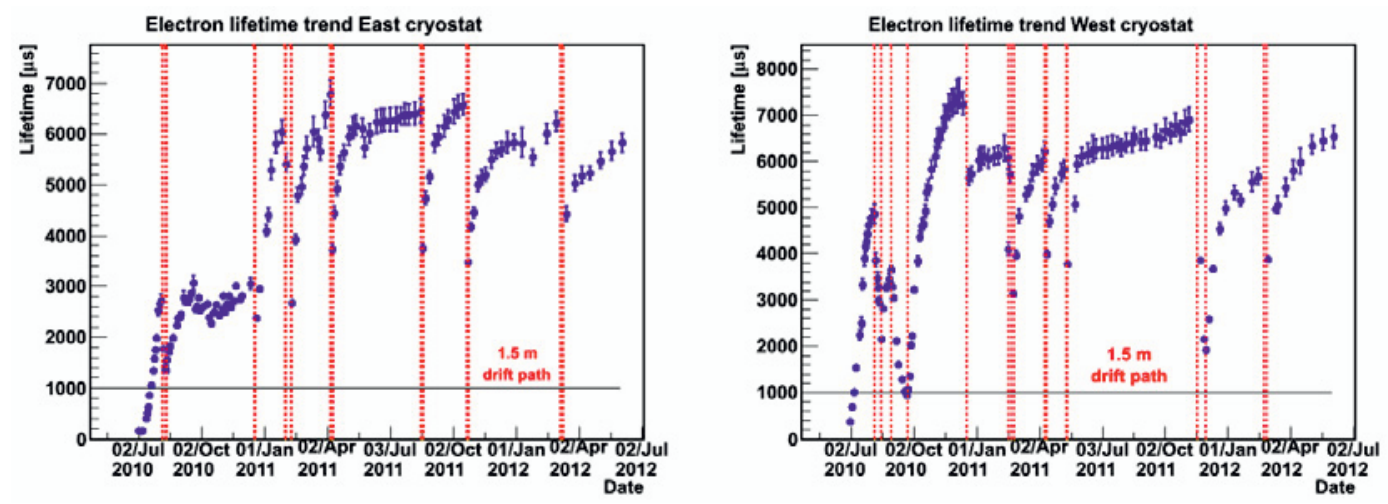

\footnotetext{
${ }^{2}$ This is due to various reactions involving different types of impurities, the most harmful of which being Oxygen [9]; moreover, LAr can be contaminated inside the cryostat from outgassing of the walls and TPC components (electrodes, cables, PMTs, etc.).
} 


\section{ICARUS T600 physics potential}

\subsection{Physics runs}

ICARUS T600 started its operation in May 2010, collecting right from the beginning both cosmic rays and CNGS neutrino events.

The trigger system relies on the scintillation light signals, with a starting layout based, for each of the four TPC chambers, on the analog sum of signal from PMTs with a 100 photoelectron discrimination low threshold.

The trigger for cosmic rays exploits the coincidence of the PMTs sum signals of the two adjacent chambers in the same half-module, relying on the $50 \%$ transparency of the cathode mechanical structure: this allows for an efficient reduction of the spurious signals maximizing the detection of low energy events. An overall acquisition rate of $\sim 35 \mathrm{mHz}$ has been achieved well below the maximum allowed DAQ rate, resulting in about $\sim 125$ cosmic events per hour.

For CNGS neutrino events the proton extraction time information is also available, since an "early warning" signal is sent from CERN to LNGS $80 \mathrm{~ms}$ before the first proton spill extraction. Thus, accounting for the CNGS SPS cycle structure, i.e. two spills $50 \mathrm{~ms}$ apart and lasting $10.5 \mu \mathrm{s}$ each, a dedicated trigger strategy has been chosen for the CNGS neutrino interactions, based on the presence of the PMT signal within a $\sim 60 \mu$ s gate opened in correspondence to the predicted extraction times delayed by the neutrino time of flight ( $2.44 \mathrm{~ms}$ ) from CERN to LNGS.

A trigger rate of about $1 \mathrm{mHz}$ is obtained, including neutrino interactions inside the detector and muons from neutrino interactions in the upstream rocks.

ICARUS CNGS run started in stable conditions on October $1^{\text {st }} 2010$, collecting $5.8 \times 10^{18}$ protons on target (pot) out of the $8 \times 10^{18}$ delivered by CERN up to November $22^{\text {nd }}$. During the 2011 run $4.44 \times 10^{19}$ pot over the $4.78 \times 10^{19}$ pot delivered by CERN has been collected, with detector live-time of $93 \%$. Data taking with CNGS beam resumed on 2012, March $23^{\text {rd }}$, and is smoothly proceeding, collecting $2.1 \times 10^{19}$ pot up to now with almost $94 \%$ detector live-time. The first $3.3 \times 10^{19}$ pot of the 2010-2011 data sample have been processed, resulting in 1091 neutrino interactions with vertex in 433 tons LAr fiducial volume, in good agreement with MC expectation.

Data collected in 2010 and part of 2011 from CNGS beam were exploited to contribute to the solution of the super-luminal neutrino problem. A different dedicate low intensity bunched beam has been used in the end of 2011 to perform the neutrino time of flight measurements. This activity was motivated by the OPERA collaboration first claim that CNGS muon neutrinos arrive to Gran Sasso, after covering a distance of about $732 \mathrm{~km}$, earlier than expected from luminal speed. The ICARUS analysis focused on two different issue: a search for the analogue to Cherenkov radiation by neutrinos at super-luminal speeds and the direct measurement of the neutrino time-of-flight.

\subsection{Observation and reconstruction of neutrino events}

The T600 at LNGS is detecting neutrino interaction events both from the CNGS beam and from cosmics.

The neutrino interaction events are then fully 3D reconstructed ${ }^{3}$ : as stated before, muons, pions, protons and kaons are identified by studying the event topology and the energy deposition per track length unit as a function of the particle range ( $d E / d x$ versus range) with a dedicated reconstruction program based on the polygonal line algorithm and on neural network. Electrons are recognized by the characteristic electromagnetic showering. Momentum of long muon tracks escaping the detector

\footnotetext{
${ }^{3} 3 \mathrm{D}$ track reconstruction starts from a 2D track finding algorithm based on an automatic clustering over an angle-position matrix; an approach based on principal curve analysis has been developed for three dimensional reconstruction.
} 
is determined by multiple scattering.

An example of a $v_{\mu} \mathrm{CC}$ candidate real data event is shown in Fig. 4. The long muon track, about 13 meters, is impressive. The leading muon deposits by ionization a total energy of about $2.7 \mathrm{GeV}$ before exiting the detector. The total hadronic energy in the event, $\mathrm{E}_{h}=2.3 \pm 0.5 \mathrm{GeV}$, is certainly underestimated, since one particle from the secondary vertex escapes from the bottom side of the detector, and neutrons may have been produced and escaped detection. The reconstructed total energy is $12.6 \pm 1.2 \mathrm{GeV}$, within the energy range of the CNGS beam; the missing transverse momentum $\simeq 250 \mathrm{MeV} / \mathrm{c}$ is consistent with the theoretical expectation from Fermi motion of target nucleon.

Figure 4. An example of $v_{\mu} \mathrm{CC}$ interaction from the CNGS beam in the ICARUS T600 detector: 1) muon from the CC conversion; 2) electromagnetic showers from $\pi_{0}$ decay; 3 ) pion; 4) multiple tracks from secondary vertex; 5) muon; 6) kaon; 7) proton; 8) escaping particle.

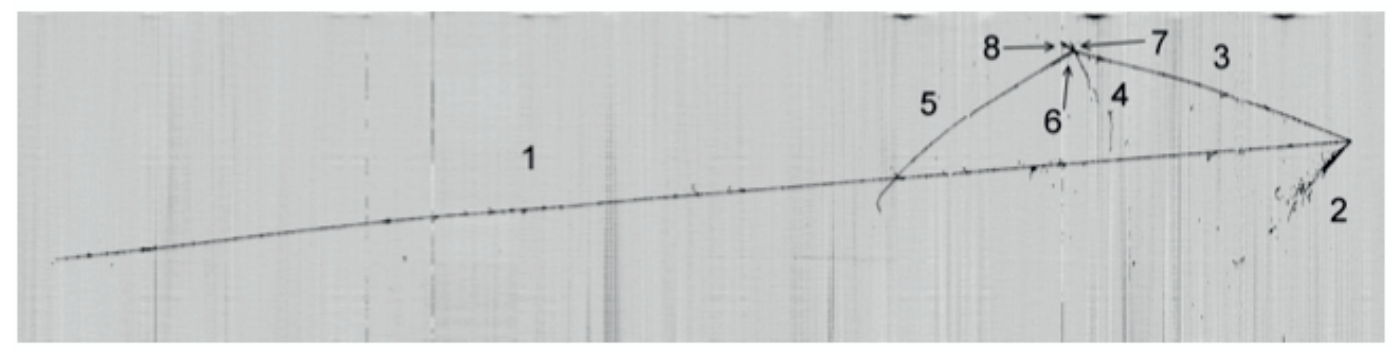

\subsection{Search for the analogue to Cherenkov radiation by neutrinos at super-luminal speeds}

Super-luminal muon neutrinos should lose their energy by producing photons and $\mathrm{e}^{+} \mathrm{e}^{-}$ bremsstrahlung pairs, through $\mathrm{Z}^{0}$ mediated processes analogous to Cherenkov radiation, as argued by Cohen and Glashow [1]. The pairs emission rate and neutrino energy loss $\mathrm{dE} / \mathrm{dx}$ are both proportional to $\delta^{3}$ where $\delta=\left(v_{v}^{2}-c^{2}\right) / c^{2}$, being $v_{v}$ and $\mathrm{c}$ the neutrino and light velocity, respectively. The OPERA result corresponds to the value of $\delta \simeq 5 \times 10^{-5}$ with small variations over the detected neutrinos energy domain.

About $1.53 \times 10^{19}$ pot were used for the analysis. The measured raw energy deposition $\mathrm{E}_{d e p}$ for CC and NC muon neutrino events was obtained from a calorimetric measurement corrected for signal quenching. The experimental distribution was compared with a full Fluka Monte Carlo simulation of the neutrino propagation to Gran Sasso for two values of $\delta$, namely $\delta=0$, and $\delta=5 \times 10^{-5}$. The experimental spectrum agrees very well with the simulations for the unaffected CERN neutrino beam (Fig. 5) and is not compatible with the spectrum resulting from the OPERA (later revised) claim. In addition, no candidate of $\mathrm{e}^{+} \mathrm{e}^{-}$pair event was found following the bremsstrahlung production criteria: this lack of events was translated into $90 \%$ CL limit of $\delta<2.5 \times 10^{-8}$ for multi-GeV neutrinos [1].

\subsection{Direct measurement of the neutrino time-of-flight}

The measurement was carried out from October $31^{\text {st }}$ to November $6^{\text {th }} 2011$, with the use of very tightly bunched, low intensity, beam with four $\simeq 3 \mathrm{~ns}$ extractions separated by $524 \mathrm{~ns}$. Seven beam-associated events were recorded during this period: this number is consistent with the CNGS delivered neutrino flux $\left(2.2 \times 10^{16}\right.$ pot $)$ and the ICARUS T600 detection efficiency. The sample consists of two CC and one NC with the vertex contained within the detector active volume, whereas four events represent 
Figure 5. Neutrino energy deposition in ICARUS for $v_{\mu} \mathrm{e} \bar{v}_{\mu}$ CC events [Left] and for for neutral current events [Right] compared with Monte Carlo expectation.
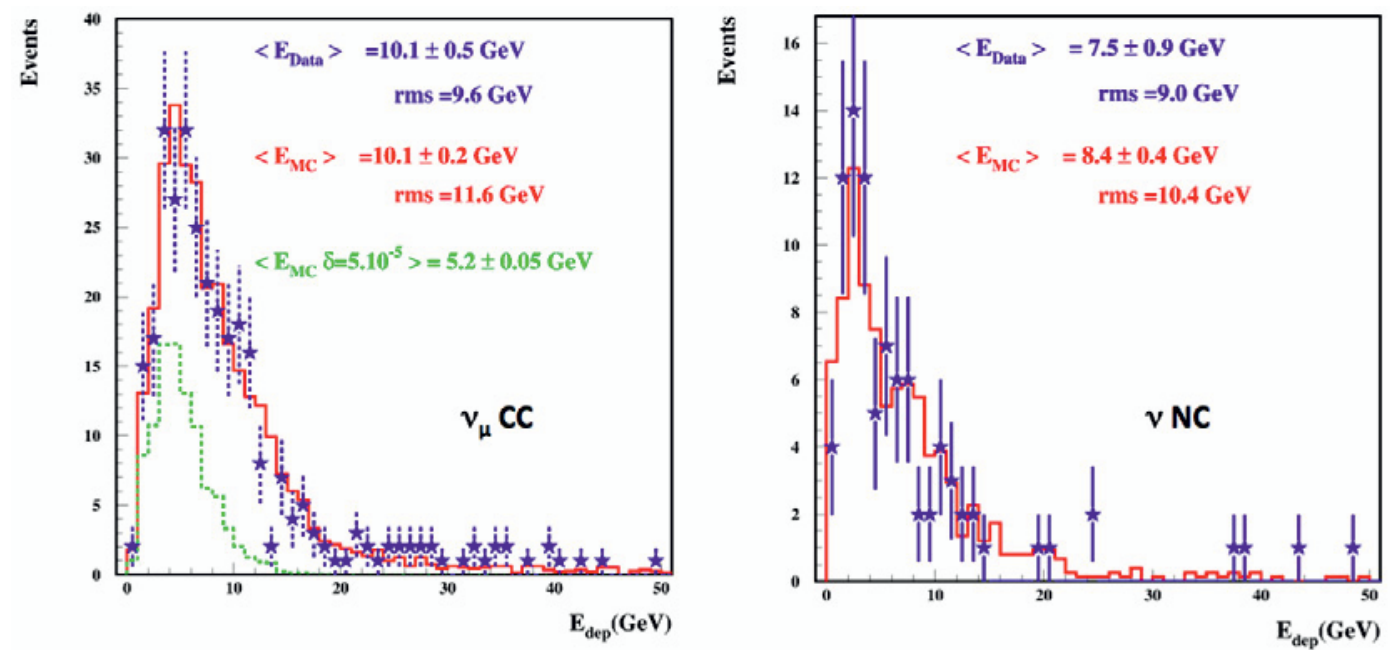

muons coming from neutrino interactions with the upstream rock. For each event the actual neutrino time of flight was obtained from to $f_{v}=T_{\text {stop }}-T_{\text {start }}$, where $T_{\text {stop }}$ is the event time in ICARUS T600 corrected for the PMT and vertex positions, $T_{\text {start }}$ is the proton transit time at the SPS/CNGS beamcurrent-transformer (BCT) accounting for the additional time related to the nearest proton bunch.

The resulting time of flight difference $\delta t=t o f_{c}-t o f_{v}$ between the speed of light and the arriving neutrino is $\delta t=\left(-0.3 \pm 4.9_{(\text {stat. }} \pm 9.0_{(\text {syst. }}\right)$ ns [1] in agreement with Lorentz dependent velocities of neutrinos and of light ( Fig. 6 [Left]); this result is not compatible with $\delta t=\left(57.8 \pm 7.8(\text { stat. })_{-5.9}^{+8.3}(\right.$ syst. $\left.)\right) \mathrm{ns}$ reported by first claim from the OPERA collaboration [1].

This measurement has been recently perfected with a data sample collected during May 2012, when the CERN-CNGS neutrino beam was operated for two weeks for a total of $1.8 \times 10^{17}$ pot in bunched mode, with a $\sim 3$ ns narrow width proton beam bunches, separated by $100 \mathrm{~ns}$. Both the ICARUS-T600 PMT-DAQ and the CERN-LNGS timing synchronization have been substantially improved for this campaign, taking advantage of additional independent GPS receivers, both at CERN and LNGS as well as of the deployment of the "White Rabbit" protocol both at CERN and LNGS.

The ICARUS-T600 detector has measured the neutrino time of flight with 25 beam-associated events, using all different available time synchronization paths.

The final result $\delta t=t o f_{c}-t o f_{v}=\left(0.18 \pm 0.69_{(\text {stat. }} \pm 2.17_{(\text {syst. }}\right) \mathrm{ns}[1]$ is in agreement with the value previously reported but with improved statistical and systematic errors (Fig. 6 [Right]).

\subsection{New perspectives for the T600 Liquid Argon TPC}

The recent observations of an electron excess in a $\bar{v}_{\mu}$ beam, made by the LSND and MiniBooNE experiments, and of an apparent disappearance signal in the $\bar{v}_{e}$ events collected by the reactor neutrino experiments, seem to suggest the presence of a new invisible "sterile" neutrino type.

Thanks to its features, LAr-TPC represents an ideal device to try to solve all this anomaly. A new experimental search with an intense $\sim 2 \mathrm{GeV}$ neutrino beam at CERN/SPS is proposed in order to definitely clarify the possible existence of additional neutrino state [1]. It is based on two strictly iden- 
Figure 6. Time of flight difference between the speed of light and the arriving neutrino at LNGS: first measurements [Left] and repeated precision measurements [Right].
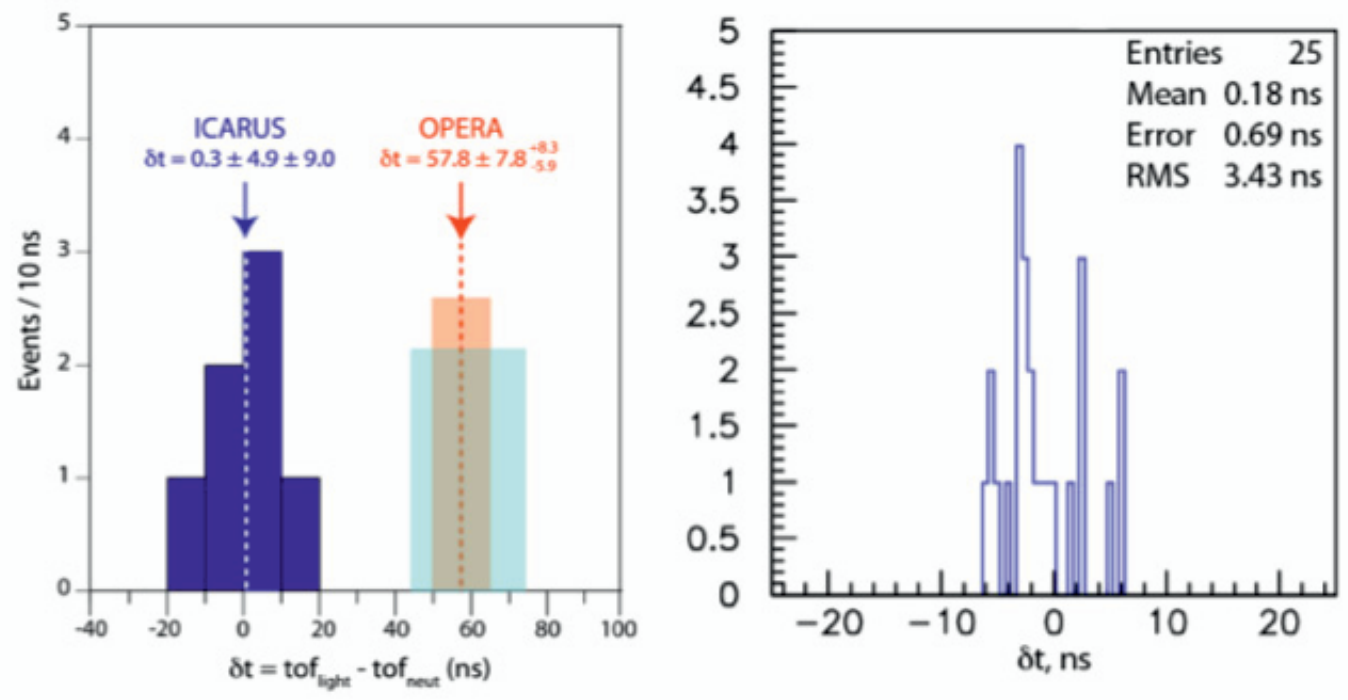

tical LAr-TPC detectors complemented by magnetized spectrometers detecting electron and muon neutrino events at Far $(1600 \mathrm{~m})$ and Near $(300 \mathrm{~m})$ positions w.r.t. the proton target: respectively ICARUS-T600 detector, to be moved to CERN after its exploitation at LNGS, and an additional new $150 \mathrm{t}$ detector to be built.

\section{Conclusions}

ICARUS T600 is the first large Liquid Argon Time Projection Chamber ( 600 tons of LAr) operated underground at INFN LNGS laboratory and addressed to the study of "rare events" and, among these, neutrino interactions.

The T600 is acquiring data without interruption since mid 2010 at LNGS with CNGS beam from CERN-SPS, searching for the $v_{\mu} \rightarrow v_{\tau}$ oscillation and LSND-like $v_{e}$ excess, as well as for atmospheric $\nu$ 's and proton decay. Its unique imaging capability, spatial/calorimetric resolutions and e $/ \pi^{0}$ separation allow to reconstruct/identify events in a new way, w.r.t. previous/current experiments; for these reasons ICARUS T600 represents a major milestone towards the realization of future LAr detectors for neutrino physics and for the search of rare events.

A very strong contribution to the super-luminal neutrino problem has been produced with the data collected in the 2011. In a dedicated search, no significant deformation of the neutrino energy spectrum and no superluminal Cherenkov-like $\mathrm{e}^{+} \mathrm{e}^{-}$pair or $\gamma$ emission event has been directly observed inside the fiducial volume of the ICARUS TPC-LAr detector.

Direct measurements of the neutrino velocity with the ICARUS detector at the CNGS beam have been performed. The first result of $\delta t=\left(-0.3 \pm 4.9_{(\text {stat. })} \pm 9.0_{(\text {syst. })}\right)$ ns gave a value compatible with the simultaneous arrival of all events with speed equal to that of light. Precision measurements operated during May 2012 with the CERN-CNGS neutrino beam in bunched mode for a total of $1.8 \times 10^{17}$ pot provided a result of $\delta t=t o f_{c}-t o f_{v}=\left(0.18 \pm 0.69_{(\text {stat. })} \pm 2.17_{(s y s t .)}\right) \mathrm{ns}$ in good agreement with the 
previous value, showing also in this case that the measured neutrino time of flight is compatible with the arrival of all events with speed equivalent to the one of light.

\section{References}

[1] C. Rubbia, CERN-EP/77-08 (1977).

[2] P. Benetti et al., Nucl. Instr. and Meth. A 332, (1993) 395.

[3] P. Cennini et al., Nucl. Instr. and Meth. A 345, (1994) 230.

[4] P. Cennini et al, Nucl. Instr. and Meth. A 432, (1999) 240-248.

[5] F. Arneodo et al., Nucl. Instr. and Meth. A 449, (2000) 36.

[6] A. Ankowski et al., Acta Phys. Polon. B41, (2010) 103.

[7] A. Ankowski et al., Eur. Phys. J. C48, (2006) 667.

[8] S. Amerio et al., Nucl. Instr. and Meth A 527, (2004) 329-410.

[9] A. Bettini et al., Nucl. Instr. and Meth. A 305, (1991) 177.

[10] P. Benetti et al., Nucl. Instr. and Meth. A 333, (1993) 567.

[11] G. Bakale et al., J. Chem. Phys. 80, (1976) 2556.

[12] A. G. Cohen and S.L. Glashow, Phys. Rev. Lett. 107, (2011) 181803.

[13] M. Antonello et al., Physics Letters B 711, (2012) 270-275.

[14] M. Antonello et al., Physics Letters B 713, (2012) 17-22.

[15] T. Adam et al., arXiv:1109.4897v2, (2011).

[16] M. Antonello et al., arXiv/1208.2629, (2012).

[17] M. Antonello et al., arXiv/1208.0862, (2012). 